\title{
Reclassification of Paenibacillus larvae subsp. pulvifaciens and Paenibacillus larvae subsp. larvae as Paenibacillus larvae without subspecies differentiation
}

Correspondence
Elke Genersch
elke.genersch@rz.hu-berlin.de

\author{
Elke Genersch, ${ }^{1}$ Eva Forsgren, ${ }^{2}$ Jaana Pentikäinen, ${ }^{3}$ Ainura Ashiralieva, ${ }^{1}$ \\ Sandra Rauch, ${ }^{1}$ Jochen Kilwinski ${ }^{4}$ and Ingemar Fries ${ }^{2}$
1Department of Molecular Microbiology, Institute for Bee Research, Friedrich-Engels-Str. 32, D-16540 Hohen Neuendorf, Germany
${ }^{2}$ Department of Entomology, Swedish University of Agricultural Sciences, Box 7044, SE-75007 Uppsala, Sweden
${ }^{3}$ National Veterinary and Food Research Institute, PL 92, FIN-70701 Kuopio, Finland
${ }^{4}$ Staatliches Veterinäruntersuchungsamt Arnsberg, Zur Taubeneiche 10-12, D-59821 Arnsberg, Germany

\begin{abstract}
A polyphasic taxonomic study of the two subspecies of Paenibacillus larvae, Paenibacillus larvae subsp. larvae and Paenibacillus larvae subsp. pulvifaciens, supported the reclassification of the subspecies into one species, Paenibacillus larvae, without subspecies separation. Our conclusions are based on the analysis of six reference strains of $P$. larvae subsp. pulvifaciens and three reference strains and 44 field isolates of $P$. larvae. subsp. larvae. The latter originated from brood or honey of clinically diseased honey bee colonies or from honey of both clinically diseased and asymptomatic colonies from Sweden, Finland and Germany. Colony and spore morphology, as well as the metabolism of mannitol and salicin, did not allow a clear identification of the two subspecies and SDS-PAGE of whole-cell proteins did not support the subspecies differentiation. For genomic fingerprinting, repetitive element-PCR fingerprinting using ERIC primers and PFGE of bacterial DNA were performed. The latter method is a high-resolution DNA fingerprinting method proven to be superior to most other methods for biochemical and molecular typing and has not previously been used to characterize $P$. larvae. ERIC-PCR identified four different genotypes, while PFGE revealed two main clusters. One cluster included most of the $P$. larvae subsp. larvae field isolates, as well as all $P$. larvae subsp. pulvifaciens reference strains. The other cluster comprised the pigmented variants of $P$. larvae subsp. larvae. 16S rRNA gene sequences were determined for some strains. Finally, exposure bioassays demonstrated that reference strains of $P$. larvae subsp. pulvifaciens were pathogenic for honey bee larvae, producing symptoms similar to reference strains of $P$. larvae subsp. larvae. In comparison with the type strain for $P$. larvae subsp. larvae, ATCC $9545^{\top}$, the $P$. larvae subsp. pulvifaciens strains tested were even more virulent, since they showed a shorter $\mathrm{LT}_{100}$. An emended description of the species is given.
\end{abstract}

Published online ahead of print on 4 November 2005 as DOI 10.1099/ ijs.0.63928-0.

Abbreviations: AFB, American foulbrood; MRPs, macrorestriction profiles; rep-PCR, repetitive element-PCR fingerprinting.

The GenBank/EMBL/DDBJ accession numbers for the 16S rRNA gene sequences of Paenibacillus larvae strains 03-525, 00-1163, 02130 and 03-189 are DQ079620-DQ079623, respectively.

A table giving the CCUG accession numbers for the $P$. larvae field strains used in the study and figures showing colony and spore morphology are available as supplementary material in IJSEM Online.

\section{INTRODUCTION}

American foulbrood (AFB), a fatal bacterial disease of honey bee brood, has spread worldwide (Matheson, 1993) and is a considerable threat to beekeeping in many countries as it is highly contagious and often lethal to managed bee colonies. The clinical symptoms of AFB are typical, with the brown, viscous larval remains forming a ropy thread when drawn out with a matchstick. The decaying brood desiccates into hard scales, tightly adhering to the walls of the cells, 
consisting of millions of bacterial spores which are the infectious stage of the pathogen.

A rare condition, known as powdery scale disease of honey bee brood, was described by Katznelson (1950). He isolated a spore-forming bacterium from powdery decays of dead larvae, considered the aetiological agent of powdery scale disease. However, later he concluded that this organism was not pathogenic to honey bees (Katznelson \& Jamieson, 1951). Yet Hitchcock et al. (1979) provided evidence that the organism isolated by Katznelson (1950) is pathogenic to honey bee larvae. The acute symptoms of this disease have never been properly described, although findings of powdery scales were later reported by Gilliam \& Dunham (1978), who isolated bacterial strains phenotypically related to those isolated by Katznelson (1950).

The causative agents of AFB and powdery scale disease were originally described as Bacillus larvae (White, 1906) and Bacillus pulvifaciens (Katznelson, 1950), respectively. Both species were later transferred to the genus Paenibacillus (Ash et al., 1993). In 1996, a polyphasic taxonomic approach, including DNA-DNA binding studies, revealed high levels of similarity between the two species that did not support the classification of two separate species. However, infraspecific differences and the contrasting described pathologies led to the reclassification of $P$. larvae strains as $P$. larvae subsp. larvae and $P$. pulvifaciens strains as $P$. larvae subsp. pulvifaciens (Heyndrickx et al., 1996).

Besides differences in pathogenicity, $P$. larvae pulvifaciens is described as differing from $P$. larvae larvae by having a striking orange-pigmented colony morphology not seen with P. larvae larvae (Heyndrickx et al., 1996), by showing differences in external spore structure (Ludvik et al., 1993) and by the production of acid from mannitol, but not from salicin (Heyndrickx et al., 1996). The presence of orangepigmented colonies is considered to be one of the most obvious hallmarks of $P$. larvae pulvifaciens, often leading to the classification of such strains as subspecies pulvifaciens. However, although orange-pigmented colonies are said to be characteristic for P. larvae pulvifaciens (Heyndrickx et al., 1996), orange-pigmented colonies isolated from diseased brood with symptoms of AFB have been reported (Drobnikovà et al., 1994) and demonstrated to represent a particular genotype of $P$. larvae larvae (Neuendorf et al., 2004). Recently, a PCR method for the differentiation of the two subspecies of $P$. larvae was developed, based on molecular subtyping performed with repetitive element-PCR (rep-PCR) using ERIC primers (Alippi et al., 2004). The results of Neuendorf et al. (2004), however, suggest strongly that the rep-PCR described by Alippi et al. (2004) might be specific for a certain genotype of $P$. larvae rather than being subspecies-specific.

There are several studies on the relatedness and classification of $P$. larvae larvae and P. larvae pulvifaciens, most of them using strain DSM $3615^{\mathrm{T}}$ or synonymous strains as a reference strain for P. larvae pulvifaciens (Alippi et al., 2002; Ash et al., 1993; Heyndrickx et al., 1996; Lauro et al., 2003). As this isolate has been demonstrated to be indistinguishable from reference strains of $P$. larvae larvae (Kilwinski et al., 2004), the validity of these studies can be questioned.

The aim of this study was to establish whether the classification of two subspecies within the species $P$. larvae is justified.

\section{METHODS}

Bacterial isolates. Forty-four field isolates of $P$. larvae from Sweden, Finland and Germany were studied (Table 1). Supplementary Table S1 (available in IJSEM Online) details the 39 strains that have been deposited at the Swedish Culture Collection, University of Gothenburg, Sweden (CCUG). Two German field isolates, 03-522 ger and 03-525 ger, were deposited at the DSMZ as DSM 16115 and DSM 16116, respectively. P. larvae larvae strains CCUG $28515^{\mathrm{T}}$, ATCC $9545^{\mathrm{T}}$ and DSM $7030^{\mathrm{T}}$ were used as reference strains. The $P$. larvae pulvifaciens reference strains used in this study were CCUG 7427 (=NCDO 1121), ATCC $49843^{\mathrm{T}}$, DSM $3615^{\mathrm{T}}$, LMG $6911^{\mathrm{T}}$, LMG 16247 (=Katznelson 754=NRRL B-3687) and LMG 16252 (=Gilliam $2=$ NRRL NRS-1684) (Table 1).

Isolation and identification. Swedish material from larvae with acute symptoms of AFB was cultivated on MYPGP-agar plates as described by Nordström \& Fries (1995). MYPGP-agar contained $\left(\mathrm{l}^{-1}\right), 10 \mathrm{~g}$ Müller-Hinton broth, $15 \mathrm{~g}$ yeast extract, $3 \mathrm{~g} \mathrm{~K}_{2} \mathrm{HPO}_{4}$, $1 \mathrm{~g}$ sodium pyruvate, $20 \mathrm{~g}$ agar and $2 \mathrm{~g}$ glucose. Finnish larvae samples and undiluted honey samples were plated on to T-HCl-YGP agar plates (Steinkraus \& Morse, 1996). T-HCl-YGP agar contained $\left(\mathrm{l}^{-1}\right), 15 \mathrm{~g}$ yeast extract (Difco), $1 \mathrm{~g}$ pyruvic acid (Sigma), $200 \mathrm{ml}$ $0 \cdot 1 \mathrm{M}$ Tris/ $\mathrm{HCl}, \mathrm{pH} 7 \cdot 0,20 \mathrm{~g}$ agar, $40 \mathrm{ml} 10 \%$ glucose and $0.6 \mathrm{ml}$ malidixic acid. Honey was boiled for $15 \mathrm{~min}$ before culturing. Plates were incubated at $35{ }^{\circ} \mathrm{C}$ for 10 days. German samples were processed as described by Neuendorf et al. (2004).

Colonies with a $P$. larvae-like morphology (whitish, somewhat transparent and glistening appearance or orange-brown, non-transparent appearance) were further analysed for catalase activity, mannitol fermentation and/or formation of giant whips. For the catalase test, part of the colony was transferred to a microscope slide and mixed with a drop of $30 \% \mathrm{H}_{2} \mathrm{O}_{2}$. Production of bubbles is indicative for catalase activity, absence of bubbles indicates lack of activity. Strains were tested by the method of Gordon et al. (1973) for the production of acid from mannitol and/or by the method of Plagemann (1985) for the formation of giant whips. In addition, all but three field isolates have already been classified by the CCUG according to colony morphology, catalase activity and API tests (Heyndrickx et al., 1996). Thirteen field isolates, some of them isolated from diseased brood showing clinical symptoms of AFB, have been preliminarily diagnosed as P. larvae pulvifaciens (Table 1).

rep-PCR analysis. Preparation of bacterial DNA for fingerprinting and subsequent rep-PCR analysis was performed essentially as previously described (Genersch \& Otten, 2003). In brief, P. larvae larvae DNA suitable for rep-PCR DNA fingerprinting was isolated from colonies grown on culture plates using $6 \%$ InstaGene matrix (BioRad) following the manufacturer's instructions. The DNA sequences of the primers used for DNA fingerprinting were $5^{\prime}$-ATGTAAGCTCCTGGGGATTCAC-3' (ERIC1R) and 5'-AAGTAAGTGACTGGGGTGAGCG-3' (ERIC2) (Versalovic et al., 1994).

PCR were carried out in a final volume of $25 \mu \mathrm{l}$ consisting of $1 \times$ reaction buffer (Qiagen) and final concentrations of $2.5 \mathrm{mM} \mathrm{MgCl}_{2}$, $250 \mu \mathrm{M}$ dNTPs (dATP, dCTP, dGTP, dTTP), $10 \mu \mathrm{M}$ primer and $0 \cdot 3 \mathrm{U}$ 
Table 1. $P$. larvae strains and overview of methods applied for characterization

ERIC, group identified with rep-PCR with ERIC primers; PFGE, NotI restriction followed by PFGE; 16S rRNA, 16S rRNA gene sequencing; PAGE, protein profiling via SDS-PAGE; Man/Sal, metabolism of mannitol and salicin as part of API tests; API ZYM, API ZYM tests for phosphatase activity. Methods used to characterize strains are indicated by + .

\begin{tabular}{|c|c|c|c|c|c|c|c|c|}
\hline Strain & Source & $\begin{array}{c}\text { Subspecies according } \\
\text { to CCUG }\end{array}$ & ERIC & PFGE & 16S rRNA & PAGE & Man/Sal & API ZYM \\
\hline DSM $7030^{\mathrm{T}}$ & DSMZ & larvae/ref. strain & I & & + & & + & \\
\hline CCUG $28515^{\mathrm{T}}$ & CCUG & larvae/ref. strain & I & & & + & & \\
\hline ATCC $49843^{\mathrm{T}}$ & ATCC & pulvifaciens/ref. strain & IV & + & & & & + \\
\hline DSM $3615^{\mathrm{T}}$ & DSMZ & pulvifaciens/ref. strain & IV & & + & & + & \\
\hline LMG $6911^{\mathrm{T}}$ & LMG & pulvifaciens/ref. strain & IV & & & & + & \\
\hline LMG 16252 & LMG & pulvifaciens/ref. strain & III & + & & + & & + \\
\hline CCUG 7427 & CCUG & pulvifaciens/ref. strain & IV & & & + & & \\
\hline 33 swe & Diseased brood & larvae & I & + & & + & + & + \\
\hline 96 swe & Diseased brood & larvae & I & + & & + & + & + \\
\hline 207/99 swe & Diseased brood & larvae & I & + & & + & + & + \\
\hline $78 / 00$ swe & Diseased brood & larvae & I & + & & + & + & + \\
\hline $166 / 00$ swe & Diseased brood & larvae & I & + & & + & + & + \\
\hline $185 / 00$ swe & Diseased brood & larvae & I & + & & + & + & + \\
\hline 233/00 swe & Diseased brood & pulvifaciens & II & + & & + & + & + \\
\hline $35 / 01$ swe & Diseased brood & larvae & I & + & & + & + & + \\
\hline $150 / 01$ swe & Diseased brood & larvae & I & + & & + & + & + \\
\hline $26 / 02$ swe & Diseased brood & pulvifaciens & II & + & & + & + & + \\
\hline $118 / 02$ swe & Diseased brood & larvae & I & + & & + & + & + \\
\hline $144 / 02$ swe & Diseased brood & larvae & I & + & & + & + & + \\
\hline $97 / 03$ swe & Diseased brood & pulvifaciens & II & + & & + & + & + \\
\hline 03-159 ger & Honey (dis. col.) & larvae & I & + & & + & + & + \\
\hline 03-189 ger & Honey (dis. col.) & & I & & + & & + & \\
\hline $03-522$ ger & Honey (dis. col.) & pulvifaciens & II & + & & + & + & + \\
\hline $03-525$ ger & Honey (dis. col.) & & II & & + & & + & \\
\hline $888 / 97$ fin & Honey & larvae & I & + & & + & + & + \\
\hline 925/97 fin & Honey & larvae & I & + & & + & + & + \\
\hline 1051/97 fin & Diseased brood & larvae & I & + & & + & + & + \\
\hline $110 / 99$ fin & Honey & larvae & I & + & & + & + & + \\
\hline $992 / 99$ fin & Honey & larvae & I & + & & + & + & + \\
\hline $910 / 01$ fin & Honey & larvae & I & + & & + & + & + \\
\hline 1275/01 fin & Honey & larvae & I & + & & + & + & + \\
\hline $1847 / 01$ fin & Diseased brood & larvae & I & + & & + & + & + \\
\hline $3264 / 02$ fin & Honey & larvae & II & + & & + & & + \\
\hline $3284 / 02$ fin & Honey & pulvifaciens & II & + & & + & + & + \\
\hline 7774/03 fin & Honey & pulvifaciens & II & & & + & + & + \\
\hline 7789/03 fin & Honey & pulvifaciens & II & + & & & + & + \\
\hline
\end{tabular}


Table 1. cont.

\begin{tabular}{|lllllllll}
\hline Strain & Source & $\begin{array}{c}\text { Subspecies according } \\
\text { to CCUG }\end{array}$ & ERIC & PFGE & 16S rRNA & PAGE & Man/Sal & API ZYM \\
\hline $7766 / 03$ fin & Honey & pulvifaciens & II & + & & & + & + \\
$8514 / 03$ fin & Honey & pulvifaciens & II & + & & + & + & + \\
$8533 / 03$ fin & Honey & pulvifaciens & II & + & & + & + \\
$8543 / 03$ fin & Honey & pulvifaciens & II & + & & + & + \\
\end{tabular}

dis. col., diseased colony with clinical symptoms.

HotStarTaq polymerase (Qiagen). The reaction conditions were as follows, after the initial activation step $\left(15 \mathrm{~min}, 95^{\circ} \mathrm{C}\right) ; 35$ cycles at $94{ }^{\circ} \mathrm{C}$ for $1 \mathrm{~min}, 53^{\circ} \mathrm{C}$ for $1 \mathrm{~min}$ and $72^{\circ} \mathrm{C}$ for $2.5 \mathrm{~min}$, followed by a final elongation step at $72{ }^{\circ} \mathrm{C}$ for $10 \mathrm{~min}$. A $5 \mu \mathrm{l}$ sample from the PCR was analysed on a $0.8 \%$ agarose gel. DNA bands were stained with ethidium bromide and visualized by UV light.

PFGE. Bacterial cells were cultured on blood agar plates for 48$72 \mathrm{~h}$ at $34^{\circ} \mathrm{C}$. A loopful of bacteria from fresh culture was suspended in $5 \mathrm{ml}$ pre-warmed $\left(37^{\circ} \mathrm{C}\right) \mathrm{LB}$ broth and incubated with shaking (150 r.p.m.) at $37^{\circ} \mathrm{C}$ for $16-18$ h. Cultures were chilled on ice and the samples were kept cold in all further steps. Depending on cell density, 2.5 or $5 \mathrm{ml}$ culture was centrifuged (4200 r.p.m., $10 \mathrm{~min}, 4{ }^{\circ} \mathrm{C}$ ) and the pellet was washed once with $1 \mathrm{ml}$ washing buffer [200 mM NaCl, $10 \mathrm{mM}$ Tris/HCl (pH 7·2), $100 \mathrm{mM}$ EDTA]. Bacterial cells were resuspended in $0.25 \mathrm{ml}$ washing buffer, quickly warmed to $37^{\circ} \mathrm{C}$, mixed with an equal amount of $1.5 \%$ InCert agarose $\left(37^{\circ} \mathrm{C}\right)$ and immediately pipetted into plug moulds. The plugs were allowed to solidify on ice and digested overnight with proteinase $\mathrm{K}$ solution $(0.5 \mathrm{M}$ EDTA $\mathrm{pH} 8.0,1 \% \mathrm{~N}$-lauroylsarcosine, $1.5 \mathrm{mg}$ proteinase $\mathrm{K} \mathrm{ml}^{-1}$ ) at $50{ }^{\circ} \mathrm{C}$. The plugs were subsequently stored in $0.5 \mathrm{mM}$ EDTA.

Before the analysis, the plugs were dialysed for 2-3 days against TEN buffer (10 mM Tris/HCl pH 7•4, $1 \mathrm{mM}$ EDTA, $50 \mathrm{mM} \mathrm{NaCl})$. The buffer was changed once during dialysis. DNA was digested with $10 \mathrm{U}$ NotI for $16 \mathrm{~h}$ at $37^{\circ} \mathrm{C}$. PFGE was performed in the Bio-Rad CHEF DR III with $1 \%$ pulsed-field certified agarose (Bio-Rad) in $0.5 \times$ Tris/ borate/EDTA buffer at $14{ }^{\circ} \mathrm{C}$. The switch time ramp was divided in two parts, $1 \cdot 0-7 \cdot 5 \mathrm{~s}$ for $17 \mathrm{~h}$ and $10 \cdot 0-30 \cdot 0 \mathrm{~s}$ for $5 \mathrm{~h}$. The switch angle was $120^{\circ}$ and the voltage was $6.0 \mathrm{~V} \mathrm{~cm}^{-1}$. After PFGE, the gels were stained with ethidium bromide and photographed under UV transillumination. Macrorestriction profiles (MRPs) were analysed visually and with the GelCompar II program (Applied Maths). One fragment difference in MRPs was interpreted as being significant. The similarity of the MRPs was determined using the Dice coefficient and clustering was performed by the unweighted pair group method with arithmetic averages (UPGMA) with a $1 \cdot 9-2 \cdot 4 \%$ tolerance.

16S rRNA gene sequencing. DNA was isolated from a loopful of bacterial cells using spin columns (NucleoSpin tissue kit; Macherey \& Nagel) in $100 \mu \mathrm{l}$ elution buffer according to the manufacturer's instructions. Extracted DNA was subjected to amplification in a final volume of $50 \mu \mathrm{l}$ containing $0 \cdot 5 \mu \mathrm{M}$ of each oligonucleotide primer (GM3F, 5'-AGAGTTTGATCMTGGC-3'; GM4R, 5'-TACCTTGTTACGACTT-3'; Buchholz-Cleven et al., 1997), $250 \mu \mathrm{M}$ (each) dATP, dCTP, dGTP and dTTP (Amersham Biosciences), $1.5 \mathrm{mM} \mathrm{MgCl}_{2}$ and $0.5 \mathrm{U}$ DNA Taq polymerase (Qiagen). PCR was performed for 29 cycles of $45 \mathrm{~s}$ at $94^{\circ} \mathrm{C}, 60 \mathrm{~s}$ at $50^{\circ} \mathrm{C}$ and $90 \mathrm{~s}$ at $72^{\circ} \mathrm{C}$.

Double-stranded PCR products were sequenced directly with the primers used for PCR using the BigDye terminator cycle sequencing kit according to the manufacturer's protocol (Applied Biosystems).
Additional primers were used to sequence upstream and downstream of the PCR product. Sequencing products were run on an ABI Prism 310 Genetic Analyzer. Sequences were edited with the OMIGA 2.0 program (Accelrys) and aligned using the CLUSTAL W algorithm (Thompson et al., 1994). The $16 \mathrm{~S}$ rRNA gene sequence data obtained from the isolates were compared with published sequences in the GenBank database using BLAST (Altschul et al., 1990).

Electron microscopy. Spores of bacterial isolates were retrieved from cultures cultivated on MYPGP agar incubated at $36{ }^{\circ} \mathrm{C}$ in an atmosphere containing $5 \% \mathrm{CO}_{2}$. Sporulating bacterial colonies were suspended in $4 \%$ glutaraldehyde (v/v) in $0.067 \mathrm{M}$ cacodylate buffer, $\mathrm{pH} 7 \cdot 4$, and kept refrigerated overnight. The spore suspension was then deposited on a polycarbonate filter $(0.6 \mu \mathrm{m}$, Nuclepore $)$ and dehydrated in a graded series of ethanol. The dehydration was completed in hexamethyldisilazane overnight. The dry filters were mounted on the sample holder by double-sided sticky tape, coated with $\mathrm{Pt} / \mathrm{Pd}$ in a sputter coater and analysed in a scanning microscope at $5 \mathrm{kV}$ (JSM 6320F; JEOL).

API. The strains were characterized biochemically using the commercially available API 50CHB and API 20E systems according to the manufacturer's instructions (bioMérieux).

API ZYM. All strains were analysed with the API ZYM test (bioMérieux). The test was performed according to the manufacturer's instructions with minor modifications. Briefly, P. larvae colonies from T-HCl-YGP agar were suspended in $3 \mathrm{ml}$ saline to obtain a turbidity of 5-6 on the McFarland scale. Each well was filled with three to four drops of the suspension. The strip was incubated at $35^{\circ} \mathrm{C}$ for $16-17 \mathrm{~h}$.

Protein profiling. SDS-PAGE was performed at the CCUG. Polyacrylamide gel electrophoresis analysis of whole-cell proteins was performed as described by Pot et al. (1994). For densitometry analysis, normalization and interpretation of protein patterns, the GelCompar software package (Applied Maths), versions 3.0 and 4.2, was used. The similarity between all pairs of traces was expressed by the Pearson product-moment correlation coefficient, which was converted to percentage similarity for convenience.

Exposure bioassay. For rearing and infecting worker larvae, we used a modified protocol according to Peng et al. (1992). Briefly, larvae were reared in 24-well tissue culture plates. The wells were either filled with $300 \mu \mathrm{l}$ larval diet (larval plate) or tightly lined with precut Kimwipes tissue (pupation plate). The grafted larvae were maintained in constant darkness in an incubator at $35^{\circ} \mathrm{C}$ and $95 \%$ relative humidity. Normal larval diet consisted of $3 \%(\mathrm{w} / \mathrm{v})$ fructose, $3 \%(\mathrm{w} / \mathrm{v})$ glucose and $66 \%(\mathrm{v} / \mathrm{v})$ royal jelly (purchased from a local bee keeper) in sterile double distilled water. For infection, the final spore concentration was adjusted using a working solution of $1 \times 10^{5}$ c.f.u. $\mathrm{ml}^{-1}$. The infectious larval diet was fed to larvae for the first $24 \mathrm{~h}$ after grafting when the larvae were between 12 and 
$36 \mathrm{~h}$ of age. Thereafter, normal larval diet was used for feeding. Control larvae were fed with normal larval diet throughout the larval stages.

Three wells in a culture plate were filled with larval diet (normal or infectious). Ten larvae of the first larval instar were grafted into each well using a special grafting tool to avoid injuring the larvae. A total of thirty larvae per plate was treated as one replicate. One experiment consisted of four replicates: three infected groups and one control group. Larvae were infected with 300 c.f.u. ml ${ }^{-1}$ (ATCC $9545^{\mathrm{T}}$ ),

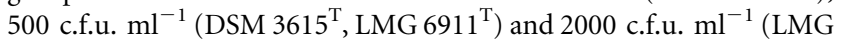
16252, LMG 16247) to obtain a mortality of at least $50 \%$. All experiments were performed at least three times.

Each day, larvae were taken out of the incubator and examined under a stereo microscope for signs of respiration, injury, disease symptoms or other abnormalities. Larvae were classified as dead when respiration ceased, they lost their body elasticity or developed marked oedema and changed colour to greyish or brownish. The number of dead larvae in each well was recorded and surviving larvae were transferred to new wells freshly filled with food. On the third, fourth, fifth and sixth day, the growing larvae were placed in groups of five, three, two and one per well, respectively. After defecation, i.e. after clear uric acid crystals and light yellow excretions could be observed in the remaining diet, engorged larvae were transferred into pupation plates. To remove adherent food, larvae were gently rolled on Kimwipes tissue before transfer. Since the larvae often continued to defecate in the pupation plates, they were transferred into clean pupation plates until defecation stopped. Engorged larvae metamorphose through a prepupal and pupal stage before emerging as adults. At all stages of development, dead animals were classified as dead from AFB only when vegetative $P$. larvae could be cultivated from the larval remains. On no occasion was $P$. larvae cultivated from the remains of dead control animals. Mortality in the control group varied from 5 to $15 \%$. Experiments with a mortality exceeding $15 \%$ in the control group were excluded, as were experiments where the 'natural' mortality (larval death but no growth of P. larvae) in the infected groups was higher than $15 \%$.

To obtain the time-course of infection, for each replicate $(n=30)$ the number of dead larvae was recorded every day. From at least three independent experiments, the mean cumulative proportion of AFBdead larvae per day \pm standard deviation was calculated and plotted against time. Survivors were excluded from this calculation (Thomas \& Elkinton, 2004). From the graph obtained, the $\mathrm{LT}_{100}$ (i.e. the time it took the pathogen to kill $100 \%$ of the infected hosts) was determined.

To evaluate whether the larval remains develop into a typical AFB scale via a ropy stage with glue-like consistency or into a powdery scale, some of the dead larvae from the infected groups were put on glass slides in Petri dishes and remained unattended in the incubator. The consistency of the larval remains during the ropy stage was tested with a matchstick. Finally, the dried-down scale was evaluated before the spores were plated on agar plates to confirm the identity of the cultivated bacterial colonies.

\section{RESULTS AND DISCUSSION}

\section{rep-PCR using ERIC primers}

A recent study suggested that analysing $P$. larvae strains by rep-PCR using ERIC primers results in two band patterns, each one specific for either $P$. larvae larvae or $P$. larvae pulvifaciens. In particular, a band of $970 \mathrm{bp}$ was considered to be specific for P. larvae larvae (Alippi et al., 2004). Genotyping of all $P$. larvae strains included in this study revealed a total of four different ERIC patterns, ERIC IERIC IV (Fig. 1). ERIC I and II showed the P. larvae larvaespecific band migrating at around 970 bp (Fig. 1, lanes 2-8), but differed in one prominent band migrating at around $2800 \mathrm{bp}$ and in one minor band migrating at around $1200 \mathrm{bp}$. Field isolates from bee hives showing the classical symptoms of AFB fell into one of these two ERIC groups. All orange-coloured $P$. larvae larvae strains belonged to the $P$. larvae larvae-specific ERIC pattern II, exhibiting an additional band migrating at 2800 bp (Fig. 1, lanes 6-8). The reference strains for $P$. larvae larvae and all field isolates not growing with pigmented colonies belonged to the $P$. larvae larvae-specific ERIC pattern I, not featuring the $2800 \mathrm{bp}$ band (Fig. 1, lanes 2-5). The reference strains for P. larvae pulvifaciens revealed two more ERIC patterns (III, IV) resembling the $P$. larvae larvae-pattern. These patterns did not possess any band migrating at around 970 bp (Fig. 1, lanes 9-13). Strain LMG 16252 was the only representative of ERIC pattern III, characterized by a double band migrating between 1500 and 2000 bp (Fig. 1, lane 9) also present in ERIC patterns I and II. Strains ATCC $49843^{\mathrm{T}}$, LMG 16247, LMG $6911^{\mathrm{T}}$ and DSM $3615^{\mathrm{T}}$ belonged to group IV, characterized by having no bands migrating more slowly than 1250 bp (Fig. 1, lanes 10-13). Although Alippi et al. (2004) described only one ERIC pattern for P. larvae larvae, the existence of the ERIC I and ERIC II patterns is consistent with an earlier report describing two ERIC patterns among German field isolates which correlated with the two patterns obtained by the same method using MBO REP1 primers (Genersch \& Otten, 2003). The two other ERIC patterns,

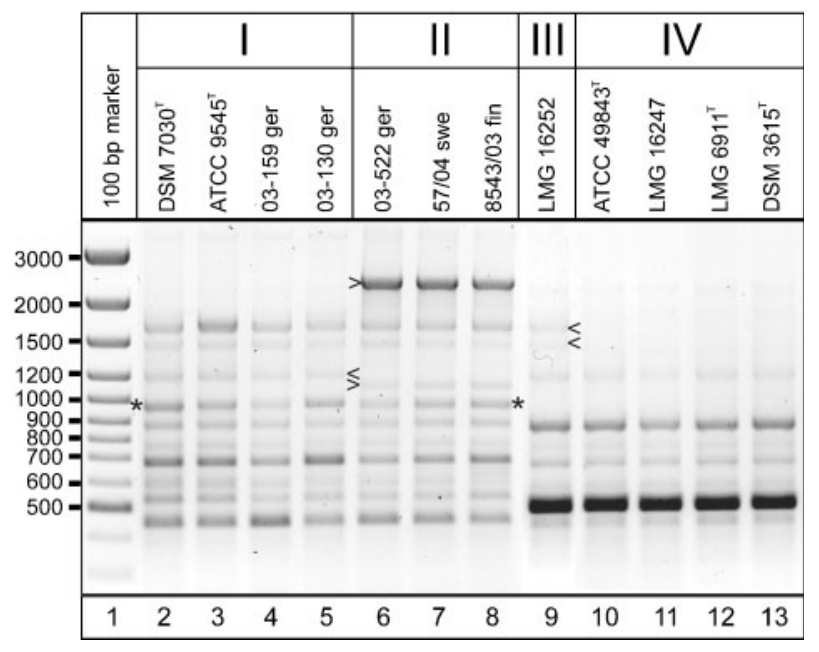

Fig. 1. Genotyping via rep-PCR using ERIC primers. Using the primer pair ERIC1R/ERIC2, field isolates and reference strains of $P$. larvae larvae (DSM $7030^{\top}$, ATCC $9545^{\top}$ ) as well as reference strains for $P$. larvae pulvifaciens (LMG 16252, ATCC 49843 ${ }^{\top}$, LMG 16247, LMG $6911^{\top}$, DSM 3615 ${ }^{\top}$ ) were analysed. The band migrating at $970 \mathrm{bp}$, considered specific for the subspecies $P$. larvae larvae, is indicated by a star. Bands important for the differentiation of the four ERIC genotypes are highlighted by arrowheads. 
ERIC III and ERIC IV, obtained for the reference strains of $P$. larvae pulvifaciens lack the 970 bp band, suggesting that this feature could be indicative for P. larvae pulvifaciens strains.

\section{PFGE}

Applying PFGE, the gold standard for genotyping (Olive \& Bean, 1999), revealed that the MRPs of $P$. larvae strains were quite similar ( $77 \%$ overall similarity) and could be divided into two main clusters (Fig. 2). One cluster contained all isolates belonging to the P. larvae genotypes ERIC I (nonpigmented field isolates as well as $P$. larvae larvae type strain ATCC $9545^{\mathrm{T}}$ ), ERIC IV (P. larvae pulvifaciens reference strains LMG 16247 and ATCC $49843^{\mathrm{T}}$ ) and ERIC III (redpigmented $P$. larvae pulvifaciens reference strain LMG 16252). The MRPs of the three P. larvae pulvifaciens reference strains were very similar, but not identical and they formed one group that differed in many ways from the profile of the typical $P$. larvae larvae strains belonging to the

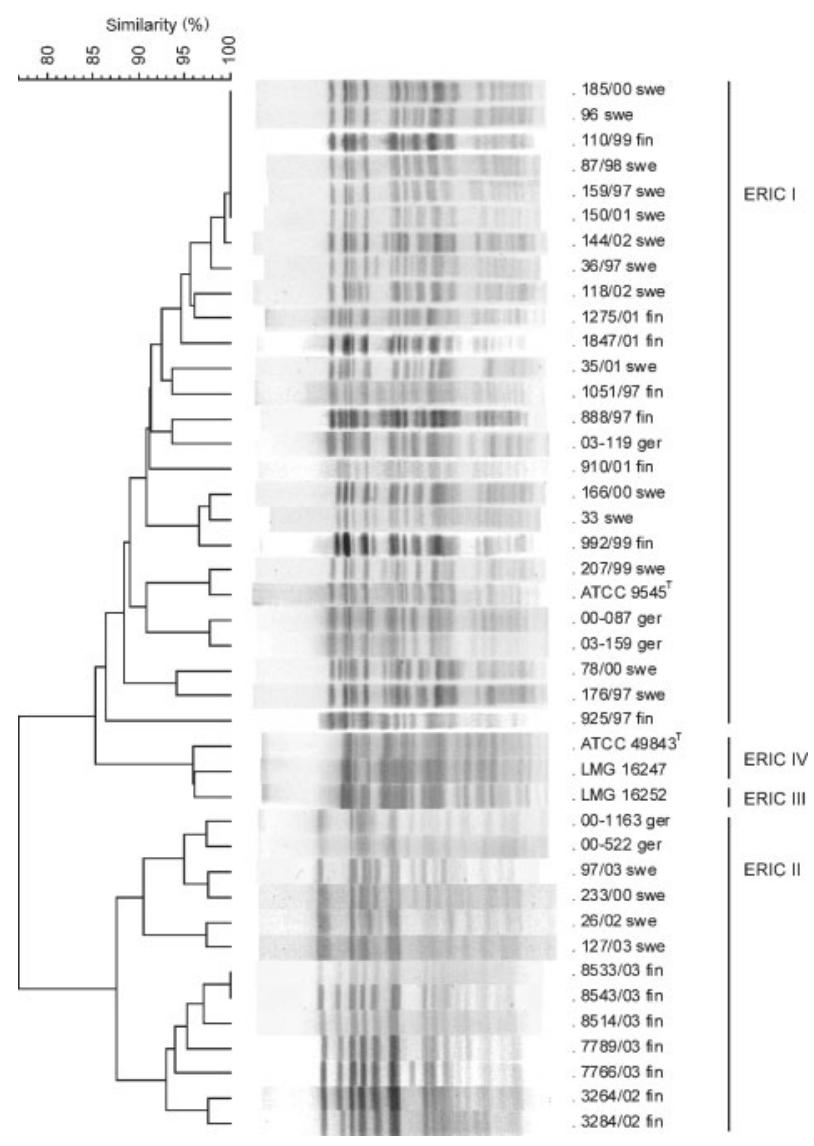

Fig. 2. Dendrogram based on the UPGMA clustering of normalized computer profiles of a PFGE analysis of $P$. larvae strains. Using PFGE, Notl MRPs of $P$. larvae larvae field isolates and reference strain ATCC $9545^{\top}$, as well as of $P$. larvae pulvifaciens reference strains ATCC $49843^{\top}$, LMG 16247 and LMG 16252, were separated. The attribution of the strains to the four ERIC genotypes is indicated.
ERIC I genotype, which showed $85 \%$ internal similarity. The other main cluster contained all $P$. larvae larvae field isolates belonging to the pigmented genotype ERIC II. All of these strains had many conserved regions and the MRPs were very similar ( $88 \%$ similarity). This cluster could be divided into two groups differing in a band at $290 \mathrm{~kb}$. All Finnish strains showed a band migrating at $290 \mathrm{~kb}$ that was not found in the German and Swedish strains. Since the three $P$. larvae pulvifaciens reference strains were more closely related to the typical $P$. larvae larvae strains, including the P. larvae larvae type strain ATCC $9545^{\mathrm{T}}$, than to the pigmented $P$. larvae larvae strains belonging to the ERIC II genotype, PFGE analysis did not support the classification of the P. larvae genotypes ERIC III and ERIC IV as a separate $P$. larvae pulvifaciens subspecies.

\section{Comparative analysis of 16S rRNA gene sequences}

Comparative analysis of the almost-complete 16S rRNA gene sequences of $P$. larvae larvae field isolates 03-525 (DSM 16116), 00-1163, 02-130, 03-189 (GenBank accession numbers DQ079620, DQ079621, DQ079622, DQ079623, respectively) with sequences of $P$. larvae larvae and $P$. larvae pulvifaciens reference strains available through GenBank showed high similarity ( $>99 \cdot 5 \%)$. Over the entire sequence range analysed (1516 bp), only six positions were polymorphic (Table 2). Field isolate 03-189 showed the most divergent $16 \mathrm{~S}$ rRNA genotype, with four positions differing from the sequence of the P. larvae larvae type strain, DSM $7030^{\mathrm{T}}$ (GenBank accession number AY530294). Nevertheless, it still had $99 \cdot 7 \%$ sequence similarity. Contradictory to the subspecies classification, the sequence of the type strain of P. larvae pulvifaciens, ATCC $49843^{\mathrm{T}}$ (GenBank accession number AY030080), was more closely related to the sequence of the type strain of $P$. larvae larvae, DSM $7030^{\mathrm{T}}$, than to the sequence of 03-189, a field isolate of $P$. larvae larvae. This correlates well with a recent study demonstrating $100 \%$ similarity between the $16 \mathrm{~S}$ rRNA gene sequences of strains DSM $3615^{\mathrm{T}}$ and DSM $7030^{\mathrm{T}}$ (Kilwinski et al., 2004). Therefore, an evaluation of the relationship between $P$. larvae larvae reference strains, $P$. larvae larvae field isolates and $P$. larvae pulvifaciens reference strains, based on the $16 \mathrm{~S}$ rRNA gene sequence, strongly suggests that the isolates belong to one species which cannot be subdivided into two subspecies.

In summary, genotypic characterization of the studied isolates demonstrated no consistent differences in rep-PCR patterns, PFGE fingerprinting or DNA sequencing data that can be used to subclassify the P. larvae species.

\section{Phenotypic characterization}

In the literature, some phenotypic characteristics that discriminate $P$. larvae pulvifaciens and $P$. larvae larvae are described (Heyndrickx et al., 1996), such as colony pigmentation, spore morphology, growth in nutrient broth and the metabolism of mannitol and salicin. 
Table 2. Polymorphic positions within 16S rRNA gene sequences

Changes are indicated relative to the sequence of P. larvae larvae DSM 7030 (AY530294). Pos., position; +, positive; -, negative; ND, not determined.

\begin{tabular}{|c|c|c|c|c|c|c|c|}
\hline Strain & $\begin{array}{c}\text { GenBank } \\
\text { accession no. }\end{array}$ & $\begin{array}{c}\text { Pos. } 52 \\
\mathrm{~A} \rightarrow \mathrm{C}\end{array}$ & $\begin{array}{c}\text { Pos. } 53 \\
\mathrm{~A} \rightarrow \text { deleted }\end{array}$ & $\begin{array}{c}\text { Pos. } 75 \\
T \rightarrow \text { deleted }\end{array}$ & $\begin{array}{c}\text { Pos. } 737 \\
\mathrm{~A} \rightarrow \mathrm{G}\end{array}$ & $\begin{array}{c}\text { Pos. } 1022 \\
\mathrm{~T} \rightarrow \mathrm{C}\end{array}$ & $\begin{array}{c}\text { Pos. } 1465 \\
\mathrm{C} \rightarrow \mathrm{G}\end{array}$ \\
\hline DSM $3615^{\mathrm{T}}$ & AY530295 & - & - & - & - & - & - \\
\hline NRRL B-3555 & AY030079 & - & - & + & + & + & ND \\
\hline 03-525 (=DSM 16116) & DQ079620 & - & - & - & - & + & - \\
\hline $00-1163$ & DQ079621 & - & - & - & - & + & - \\
\hline 03-189 & DQ079623 & - & - & + & + & + & + \\
\hline
\end{tabular}

${ }^{*}$ The sequence of ATCC $49843^{\mathrm{T}}$ starts at position 49 of the DSM $7030^{\mathrm{T}}$ sequence; therefore, positions $52 / 53$ relate to positions $4 / 5$ of the ATCC $49843^{\mathrm{T}}$ sequence. Hence, these polymorphisms might be the result of sequence errors that occur frequently near the beginning of a sequencing reaction.

Among the reference strains for P. larvae pulvifaciens, strain LMG 16252 (ERIC III) grew with orange-red colonies when cultivated on sheep blood agar plates. This strain was the only one that also showed strong haemolysis on sheep blood agar plates. The other reference strains (ERIC IV) revealed a colony morphology with a whitish to greyish, somewhat transparent and slightly glistening appearance, as did the two type strains for P. larvae larvae, DSM $7030^{\mathrm{T}}$ and ATCC $9545^{\mathrm{T}}$ (ERIC I). The field isolates of $P$. larvae larvae showed both kinds of colony morphology, whitish (ERIC I) and orange-pigmented (ERIC II) (see Supplementary Fig. S1 in IJSEM Online and Table 3).

Electron microscopy revealed that spores of the $P$. larvae larvae type strain, ATCC $9545^{\mathrm{T}}$ (ERIC I), had a smooth surface while spores of an orange-pigmented $P$. larvae larvae field isolate, 233/00 swe (ERIC II), had a convoluted surface resembling the surface of a brain. In contrast, the spore surface of the P. larvae pulvifaciens type strain DSM $3615^{\mathrm{T}}$
(ERIC IV) was characterized by ridges running longitudinally along the slightly rod-shaped spores. In addition to these ridges, the surface of spores of the pigmented $P$. larvae pulvifaciens reference strain LMG 16252 (ERIC III) also showed smaller ridges lying mostly perpendicular to and between the longer ridges (see Supplementary Fig. S2 in IJSEM Online for scanning electron microscopy images of spores of all four genotypes). These results are consistent with earlier reports. While the surface of P. larvae larvae spores was described as smooth (Alippi, 1991), the spores of $P$. larvae pulvifaciens showed characteristic ridges on the surface (Ludvik et al., 1993). The convoluted surface of $P$. larvae larvae isolates belonging to the pigmented variant has not been described previously. Although P. larvae pulvifaciens has been described as having ridges running longitudinally along the rod-shaped spores, in contrast to the smooth surface of $P$. larvae larvae spores (Ludvik et al., 1993), this difference can no longer be used to distinguish between the two $P$. larvae subspecies since one of the type

Table 3. Phenotypic characteristics of $P$. larvae ERIC genotypes

All genotypes have ellipsoidal spores and are pathogenic for honey bees. Diseased larvae show clinical symptoms of AFB. Larval remains have a glue-like consistency and develop into a hard scale.

\begin{tabular}{|lcccc|}
\hline Characteristic & ERIC I & ERIC II & ERIC III & ERIC IV \\
\hline Pigmented colonies & - & + & + & - \\
Haemolysis & - & - & + & - \\
Spore surface & Smooth & Convoluted & With ridges & With ridges \\
Subculturing in nutrient broth & - & + & + & + \\
Fermentation of mannitol & - & + & + & + \\
Fermentation of salicin & + & - & + & - \\
Alkaline phosphatase & + & $\sim$ & +7 & + \\
Acid phosphatase & + & & & +7 \\
LT $_{100}$ (days) & $\sim 12$ & & & + \\
\hline
\end{tabular}

${ }^{\star}$ Data from Genersch et al., 2005. 
strains for P. larvae pulvifaciens (DSM $3615^{\mathrm{T}}$ ) containing such ridges has unambiguously been characterized as $P$. larvae larvae (Kilwinski et al., 2004).

According to Heyndrickx et al. (1996), P. larvae larvae is unable to grow in nutrient broth on repeated subculture while P. larvae pulvifaciens is able to do so. Our data revealed that growth behaviour in nutrient broth on repeated subculture depends on genotype (Table 3 ). Only $P$. larvae larvae of the ERIC I genotype was unable to grow under these conditions, while the pigmented P. larvae larvae of the ERIC II genotype and the reference strains for P. larvae pulvifaciens grew well on repeated subculture in nutrient broth. All genotypes could be subcultured on plates repeatedly if colonies were resuspended in nutrient broth before streaking them onto new agar plates.

P. larvae larvae is described as metabolizing salicin (may be weak), but not mannitol, whereas P. larvae pulvifaciens is described as being able to use mannitol but not salicin (Heyndrickx et al., 1996). Analysing all strains using the API $50 \mathrm{CHB}$ and API 20E system indeed revealed significant differences in the metabolism of these two carbon sources. But, in contrast to the results of Heyndrickx et al. (1996), in our data, subspecies allocation did not correlate with metabolic phenotype. Only the non-pigmented $P$. larvae larvae strains (ERIC I) were able to use salicin, but not mannitol (Table 3). All other strains showed the opposite characteristics and included not only the P. larvae pulvifaciens reference strains, but also the pigmented $P$. larvae larvae genotype ERIC II, suggesting that the metabolism of mannitol and salicin was genotype-specific rather than specific for the subspecies $P$. larvae larvae and $P$. larvae pulvifaciens. Further biochemical analysis using the API ZYM technique revealed differences in the activity of acid and alkaline phosphatases (Table 3). Using this method, the P. larvae pulvifaciens reference strains clustered together with the ERIC I genotype of $P$. larvae larvae. They showed a positive reaction for acid- and alkaline-phosphatases, while the pigmented $P$. larvae larvae genotype ERIC II was negative for alkaline phosphatase and showed only a weak reaction for acid phosphatase. These results confirm and expand earlier reports of genotype-specific differences in the metabolism and biochemistry of $P$. larvae larvae (Neuendorf et al., 2004), but they do not support the classification of two subspecies within the species $P$. larvae.

\section{SDS-PAGE}

Analysing the protein profiles of the $P$. larvae strains revealed two clusters (Fig. 3). One cluster comprised all but one of the pigmented P. larvae larvae field isolates (ERIC II) and the $P$. larvae pulvifaciens reference strains LMG 16252 (ERIC III), CCUG 7427 and LMG 16247 (both ERIC IV). The other cluster included all the non-pigmented field isolates and the reference strain CCUG $28515^{\mathrm{T}}$ of P. larvae

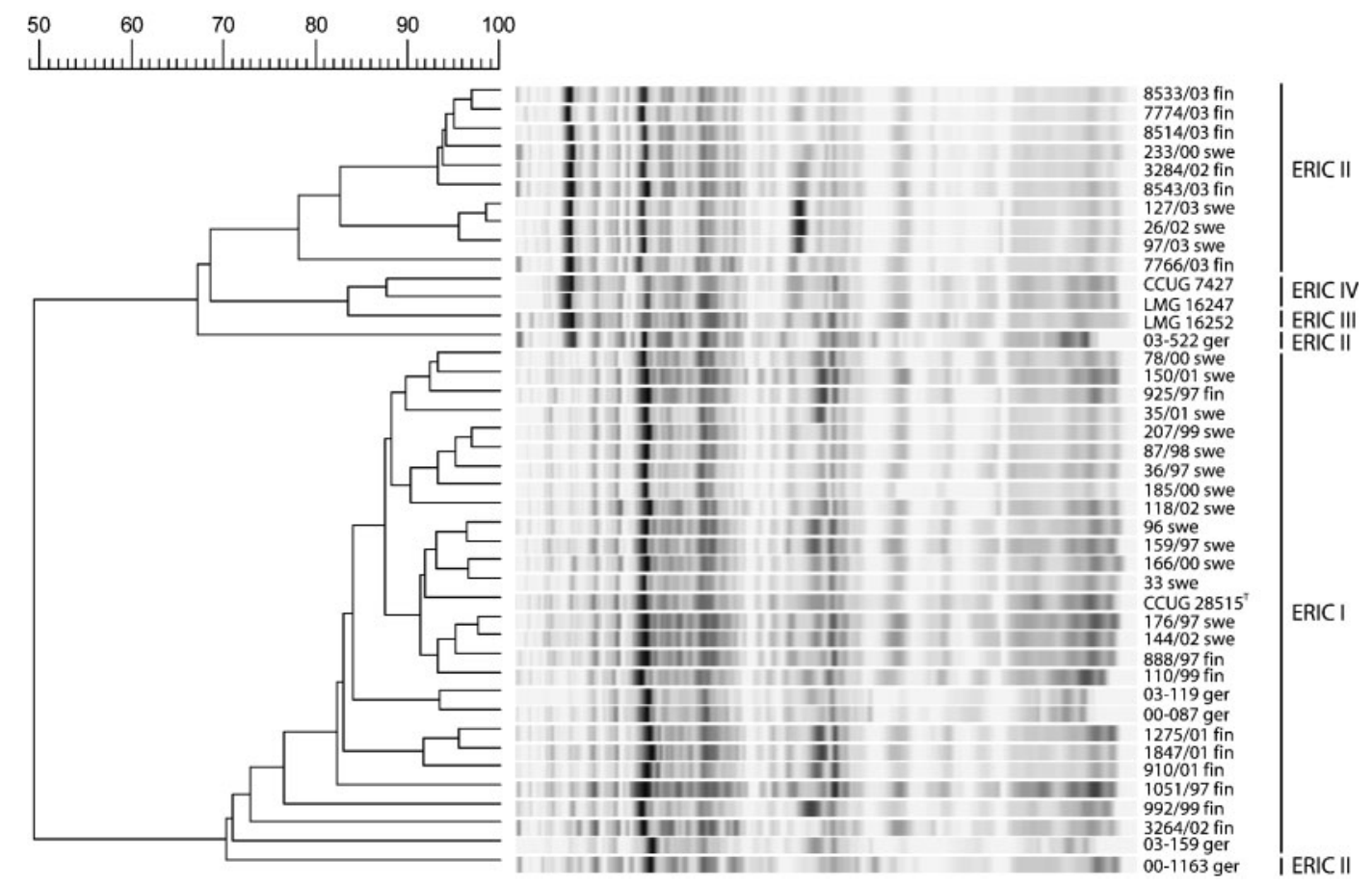

Fig. 3. Dendrogram based on the UPGMA clustering of normalized computer profiles of protein patterns (SDS-PAGE) of $P$. larvae strains. Field isolates of $P$. larvae larvae and reference strain CCUG $28515^{\top}$, as well as $P$. larvae pulvifaciens reference strains CCUG 7427, LMG 16247 and LMG 16252, were analysed by SDS-PAGE. Attribution of the strains to the four different ERIC genotypes is indicated. Bar, \% similarity. 
larvae (ERIC I), as well as one pigmented field isolate. The two clusters exhibited only $49 \%$ similarity; within each cluster the similarity was $68 \%$. Such a low clustering level within the species $P$. larvae has already been described by Heyndrickx et al. (1996). In this study, clustering above $82 \%$ could only be achieved by leaving out all bands with molecular masses of more than $1 \cdot 3 \times 10^{5}$. However, P. larvae pulvifaciens reference strains clustered separately from $P$. larvae larvae reference strains, supporting the notion of two different infraspecific groups (Heyndrickx et al., 1996). Our data reveal that the $P$. larvae pulvifaciens reference strains and some, but not all, of the pigmented $P$. larvae larvae genotype form one cluster, clearly differing from the other cluster. This confirms infraspecific differences, but does not support the classification of $P$. larvae into two separate subspecies based on current subspecies descriptions (Heyndrickx et al., 1996).

\section{Exposure bioassays}

A strong argument for the classification of $P$. larvae into two subspecies has been the different pathologies of $P$. larvae larvae and P. larvae pulvifaciens (Heyndrickx et al., 1996). $P$. larvae larvae is a highly virulent pathogen of honey bee larvae causing AFB, whereas $P$. larvae pulvifaciens is considered as an almost avirulent pathogen causing the extremely rare powdery scale disease of honey bee larvae (Katznelson, 1950; Heyndrickx et al., 1996). Exposure bioassays revealed that all representatives of the species $P$. larvae, including all reference strains for $P$. larvae pulvifaciens, were pathogenic to honey bee larvae (Table 3 ). In contrast to the situation described so far from field observations, larvae experimentally infected with $P$. larvae pulvifaciens never developed into a powdery scale, but the larval remains formed a glue-like colloid which dried down to a hard scale as described for AFB (Table 3). In addition, when the $\mathrm{LT}_{100}$ for the different strains were compared, the $P$. larvae pulvifaciens reference strains proved to be even more virulent than the P. larvae larvae type strain ATCC $9545^{\mathrm{T}}$. While it took the P. larvae pulvifaciens reference strains as little as 7 days to kill all infected larvae, it took strain ATCC $9545^{\mathrm{T}}$ around 12 days to kill $100 \%$ of the infected hosts (Fig. 4). This fast-killing phenotype is not restricted to the $P$. larvae pulvifaciens reference strains as it can also be observed for isolates belonging to the P. larvae larvae ERIC II group (Genersch et al., 2005). Therefore, different pathologies for P. larvae larvae and P. larvae pulvifaciens cannot be confirmed by exposure bioassays. At present, we have no explanation for the field observations resulting in the description of a powdery scale disease (Katznelson, 1950).

In summary, phenotypic characterization of the studied isolates demonstrates no consistent differences in spore morphology, culture characteristics, utilization of carbon sources, enzyme activity or protein profiles that can be used to differentiate between subspecies of $P$. larvae.

The data presented here support the conclusion that the reference and type strains for the subspecies $P$. larvae

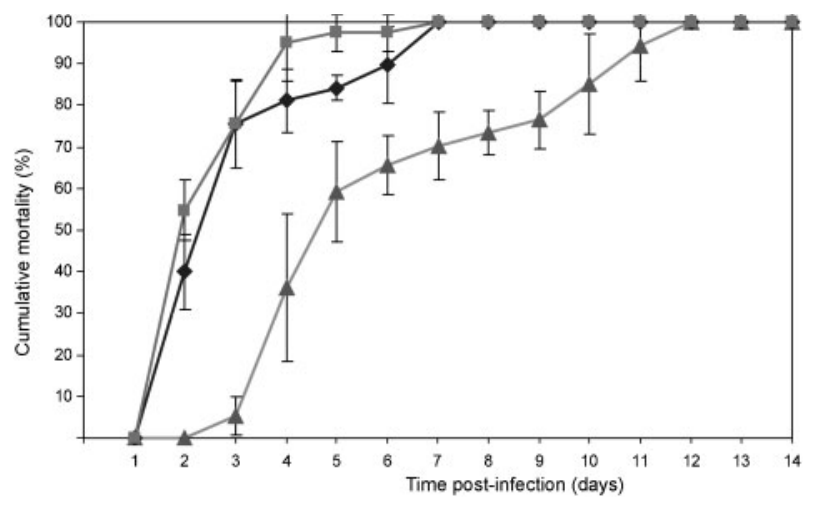

Fig. 4. Course of infection and determination of $\mathrm{LT}_{100} . P$. larvae larvae and $P$. larvae pulvifaciens reference strains were tested for pathogenicity and virulence in exposure bioassays. To determine the $\mathrm{LT}_{100}$, mortality was monitored every day and the cumulative mortality was calculated and expressed as percentage of all infected hosts, i.e. all larvae and pupae that died from AFB. Representative results obtained for three genotypes are shown. $\boldsymbol{\Delta}$, ERIC I (ATCC 9545'); ERIC III (LMG 16252); $\mathbf{\square}$, ERIC IV (DSM 3615 ${ }^{\top}$ ).

pulvifaciens represent two genotypes within the species $P$. larvae. In a polyphasic analysis comprising morphological and biochemical tests, genomic fingerprints and exposure bioassays, these genotypes always clustered together with at least one of the two other genotypes of $P$. larvae, which were unambiguously identified as $P$. larvae larvae via a subspecies-specific PCR method (Alippi et al., 2004).

As compelling evidence, based on a polyphasic approach, shows that the two subspecies represent variants of one pathogen that differs in virulence, it is proposed that both the former subspecies should be reclassified as one species, $P$. larvae. No differences between $P$. larvae pulvifaciens and $P$. larvae larvae that justify the existence of two subspecies within the species $P$. larvae could be demonstrated. In particular, no differences in pathology could be demonstrated in exposure bioassays. Thus, the OIE definitions of the causative agent of AFB in honey bees need to be amended accordingly. An emended description of $P$. larvae is given.

\section{Emended description of Paenibacillus larvae}

The morphological, chemotaxonomic and general characters of Paenibacillus larvae are as already described for this species (Heyndrickx et al., 1996). All representatives of this species are pathogenic to honey bee larvae, but differ in virulence. Decaying larvae will develop a ropy thread and a non-powdery scale. Depending on the $\mathrm{LT}_{100}$, infected or dead larvae will be removed by the nurse bees before entering the pupation stage or left decaying in the capped cell. Growth in nutrient broth on repeated subculture depends on genotype. Strains will produce non-pigmented or yellow-orange to red-pigmented colonies when cultivated 
on Columbia blood agar or MYPGP agar, depending on genotype. Different isolates may be catalase-negative or weak-delayed positive, depending on age and genotype. The type strain of the former subspecies $P$. larvae larvae, ATCC $9545^{\mathrm{T}}\left(=\mathrm{DSM} 7030^{\mathrm{T}}=\right.$ CCUG $\left.28515^{\mathrm{T}}\right)$, could now serve as the reference strain for P. larvae genotype ERIC I. Two field isolates of genotype ERIC II, 03-522ger and 03-525ger, have been deposited as DSM 16115 and DSM 16116, respectively. All Swedish and Finnish field isolates of genotype ERIC II, as well as two German isolates of genotype ERIC II, 00-1163ger and 03-522ger, have been deposited at CCUG. A table detailing all strains deposited at CCUG with the respective accession numbers is available as Supplementary Table S1 in IJSEM Online). Only one reference strain of genotype ERIC III (Gilliam $2=$ NRRL NRS-1684 = LMG 16252) is available, originating from a single isolation (Gilliam \& Dunham, 1978). Several type and reference strains for the former subspecies P. larvae pulvifaciens, ATCC $49843^{\mathrm{T}}$ (=DSM $3615^{\mathrm{T}}=$ LMG $6911^{\mathrm{T}}$ ), LMG 16247 and CCUG 7427, can now serve as reference strains for the P. larvae genotype ERIC IV.

\section{ACKNOWLEDGEMENTS}

We thank Enevold Falsen for image processing, Elisabeth Inganäs, Susanne Jensie Markopoulos, Kati Hedtke and Marion Schröder for technical assistance. E. G., A. A. and S. R. were supported by grants from the Ministries of Agriculture of Brandenburg and SachsenAnhalt.

\section{REFERENCES}

Alippi, A. M. (1991). A comparison of laboratory techniques for the detection of significant bacteria of the honey bee, Apis mellifera, in Argentina. J Apic Res 30, 75-80.

Alippi, A. M., López, A. C. \& Aguilar, O. M. (2002). Differentiation of Paenibacillus larvae subsp. larvae, the cause of American foulbrood of honeybees, by using PCR and restriction fragment analysis of genes encoding 16S rRNA. Appl Environ Microbiol 68, 3655-3660.

Alippi, A. M., López, A. C. \& Aguilar, O. M. (2004). A PCR-based method that permits specific detection of Paenibacillus larvae subsp. larvae, the cause of American foulbrood of honey bees, at subspecies level. Lett Appl Microbiol 39, 25-33.

Altschul, S. F., Gish, W., Miller, W., Myers, E. W. \& Lipman, D. J. (1990). Basic local alignment search tool. J Mol Biol 215, 403-410.

Ash, C., Priest, F. G. \& Collins, M. D. (1993). Molecular identification of rRNA group 3 bacilli (Ash, Farrow, Wallbanks and Collins) using a PCR probe test. Proposal for the creation of a new genus Paenibacillus. Antonie van Leeuwenhoek 64, 253-260.

Buchholz-Cleven, B. E. E., Rattunde, B. \& Straub, K. L. (1997). Screening for genetic diversity of isolates of anaerobic $\mathrm{Fe}$ (II)oxidizing bacteria using DGGE and whole-cell hybridization. Syst Appl Microbiol 20, 301-309.

Drobniková, V., Ritter, V., Häusler, J. \& Pytelova, I. (1994). Characterization of Bacillus larvae and related bacilli by chromatography of cell fatty acids. J Apic Res 33, 69-74.

Genersch, E. \& Otten, C. (2003). The use of repetitive element PCR fingerprinting (rep-PCR) for genetic subtyping of German field isolates of Paenibacillus larvae subsp. larvae. Apidologie 34, 195-206.
Genersch, E., Ashiralieva, A. \& Fries, I. (2005). Strain- and genotype-specific differences in virulence of Paenibacillus larvae subsp. larvae, a bacterial pathogen causing American foulbrood disease in honeybees. Appl Environ Microbiol 71, 7551-7555.

Gilliam, M. \& Dunham, R. D. (1978). Recent isolations of Bacillus pulvifaciens from powdery scales of honey bee, Apis mellifera, larvae. J Invertebr Pathol 32, 222-223.

Gordon, R. E., Haynes, W. C. \& Pang, C. H.-N. (1973). The genus Bacillus. In Handbook of Microbiology, pp. 78-81. Edited by A. I. Laskin \& H. A. Lechevalier. Cleveland, OH: Chemical Rubber Press.

Heyndrickx, M., Vandemeulebroecke, K., Hoste, B., Janssen, P., Kersters, K., De Vos, P., Logan, N. A., Ali, N. \& Berkeley, R. (1996). Reclassification of Paenibacillus (formerly Bacillus) pulvifaciens (Nakamura 1984) Ash et al. 1994, a later subjective synonym of Paenibacillus (formerly Bacillus) larvae (White 1906) Ash et al. 1994, as a subspecies of $P$. larvae, with emended descriptions of $P$. larvae as P. larvae subsp. larvae and P. larvae subsp. pulvifaciens. Int J Syst Bacteriol 46, 270-279.

Hitchcock, J. D., Stoner, A., Wilson, W. T. \& Menapace, D. M. (1979). Pathogenicity of Bacillus pulvifaciens to honey bee larvae of various ages (Hymenoptera: Apidae). J Kansas Entomol Soc 52, 238-246.

Katznelson, H. (1950). Bacillus pulvifaciens (n. sp.), an organism associated with powdery scale of honeybee larvae. J Bacteriol 59, 153-155.

Katznelson, H. \& Jamieson, C. A. (1951). Antibiotics and other chemotherapeutic agents in the control of bee diseases. Sci Agric 32, 219-225.

Kilwinski, J., Peters, M., Ashiralieva, A. \& Genersch, E. (2004). Proposal to reclassify Paenibacillus larvae subsp. pulvifaciens DSM 3615 (ATCC 49843) as Paenibacillus larvae subsp. larvae. Results of a comparative biochemical and genetic study. Vet Microbiol 104, $31-42$.

Lauro, F. M., Favaretto, M., Covolo, L., Rassu, M. \& Bertoloni, G. (2003). Rapid detection of Paenibacillus larvae from honey and hive samples with a novel nested PCR protocol. Int J Food Microbiol 81, 195-201.

Ludvik, J., Benada, O. \& Drobnikova, V. (1993). Ultrastructure of Bacillus pulvifaciens. Arch Microbiol 159, 114-118.

Matheson, A. (1993). World bee health report. Bee World 74, 176-212.

Neuendorf, S., Hedtke, K., Tangen, G. \& Genersch, E. (2004). Biochemical characterization of different genotypes of Paenibacillus larvae subsp. larvae, a honey bee bacterial pathogen. Microbiology 150, 2381-2390.

Nordström, S. \& Fries, I. (1995). A comparison of media and cultural conditions for identification of Bacillus larvae in honey. $J$ Apic Res 34, 97-103.

Olive, D. M. \& Bean, P. (1999). Principles and applications of methods for DNA-based typing of microbial organisms. J Clin Microbiol 37, 1661-1669.

Peng, Y.-S. C., Mussen, E., Fong, A., Montague, M. A. \& Tyler, T. (1992). Effects of chlortetracycline on honey bee worker larvae reared in vitro. J Invertebr Pathol 60, 127-133.

Plagemann, O. (1985). Eine einfache Kulturmethode zur bakteriologischen Identifizierung von Bacillus larvae mit Columbia-BlutSchrägagar. Berl Munch Tierärztl Wschr 98, 61-62 (in German).

Pot, B., Vandamme, P. \& Kersters, K. (1994). Analysis of electrophoretic whole-organism protein fingerprints. In Chemical Methods in Prokaryotic Systematics, pp. 493-521. Edited by M. Goodfellow \& A. G. O'Donnell. Chichester: Wiley.

Steinkraus, K. H. \& Morse, R. A. (1996). Media for the detection of Bacillus larvae spores in honey. Acta Biotechnol 16, 57-64. 
Thomas, S. R. \& Elkinton, J. S. (2004). Pathogenicity and virulence. J Invertebr Pathol 85, 146-151.

Thompson, J. D., Higgins, D. G. \& Gibson, T. J. (1994). CLUSTAL W: improving the sensitivity of progressive multiple sequence alignment through sequence weighting, position-specific gap penalties and weight matrix choice. Nucleic Acids Res 22, 4673-4680.
Versalovic, J., Schneider, M., De Bruijn, F. J. \& Lupski, J. R. (1994). Genomic fingerprinting of bacteria using repetitive sequence-based polymerase chain reaction. Methods Mol Cell Biol 5, 25-40.

White, G. F. (1906). The bacteria of the apiary with special reference to bee disease. In Bureau of Entomology, Technical Series no. 14, pp. 1-50. Washington, DC: US Department of Agriculture. 